\title{
Rare Case of Osseous Metaplasia in the Setting of Saline Breast Implantation: A Case Report
}

\section{Fan J*, Chandandeep N, Ortiz-Perez T, and Benveniste AP \\ Baylor College of Medicine, Lester and Sue Smith Breast Center, Houston, Texas, USA}

*Corresponding author: Joanna Fan, Baylor College of Medicine, Lester and Sue Smith Breast Center, 1 Baylor Plaza Houston, Texas 77030, USA, Tel: 713-798-6362;

Email: joanna.fan@bcm.edu

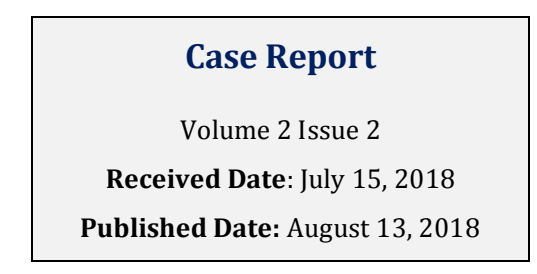

\begin{abstract}
Osseous metaplasia is rare entity with well known occurrence within various organs of the body, ranging from the paranasal sinus, thymus, endometrium, adrenal glands, colon, to the urinary bladder. It usually occurs in the setting of malignancy, but very rarely it may occur under benign conditions. Here, we present an interesting case of osseous metaplasia in a patient with only a history of saline breast implants. To our knowledge, it is the only published case of osseous metaplasia with this presentation.

Keywords: Metaplasia; Tomosynthesis; Heterotopic; Ossesous
\end{abstract}

Abbreviations: CAD: Computer Aided Detection; BIRADS: Breast Imaging-Reporting and Data System.

\section{Case Presentation}

We present an interesting and rare case of ossesous metaplasia discovered on routine screening mammographyin a patient with only a history of saline breast implantation.

A 51 year old Caucasian female, with no current breast concerns presents for routine well-woman mammographic screening to a Texas breast imaging clinic. Other than a surgical history of saline breast implantation preformed approximately 15 years ago, there is no known trauma or surgery to either breast. The patient has no other prior medical history.

A 3-dimensional bilateral digital mammogram with tomosynthesis and Computer Aided Detection (CAD) were performed per institutional protocol (per breast augmentation). Three outside mammograms were submitted for comparison, dating approximately back to 2009, 2012 and 2013.

On the routine bilateral digital mammogram with tomosynthesis, a new $2 \mathrm{~mm}$ group of calcifications at 1 o'clock posterior depth in the left breast was discovered. There were no associated overlying skin changes. Benign calcifications, but no significant masses or other findings were seen in the right breast. Scattered fibro-glandular elements were present both breasts. Patient was given BIRADS 0, indeterminate status, and she was called back for spot magnification views of the new group of calcifications.

The patient returned to outpatient imaging center approximately ten days later for left breast diagnostic mammogram imaging. Again, the $2 \mathrm{~mm}$ group of calcifications posterior depth at 1 o'clock was visualized Figures 1 \& 2. On magnifications views, they were 


\section{Clinical Radiology \& Imaging Journal}

determined to be coarse and heterogeneous. According to Bi-Rads $5^{\text {th }}$ edition atlas, group calcifications with these morphologic features are associated with $13 \%$ chance of malignancy. Accordingly, the group of new calcifications was assigned a rating of BI-RADS 4- suspicious abnormality. A stereotactic guided biopsy was recommended and performed the same day.
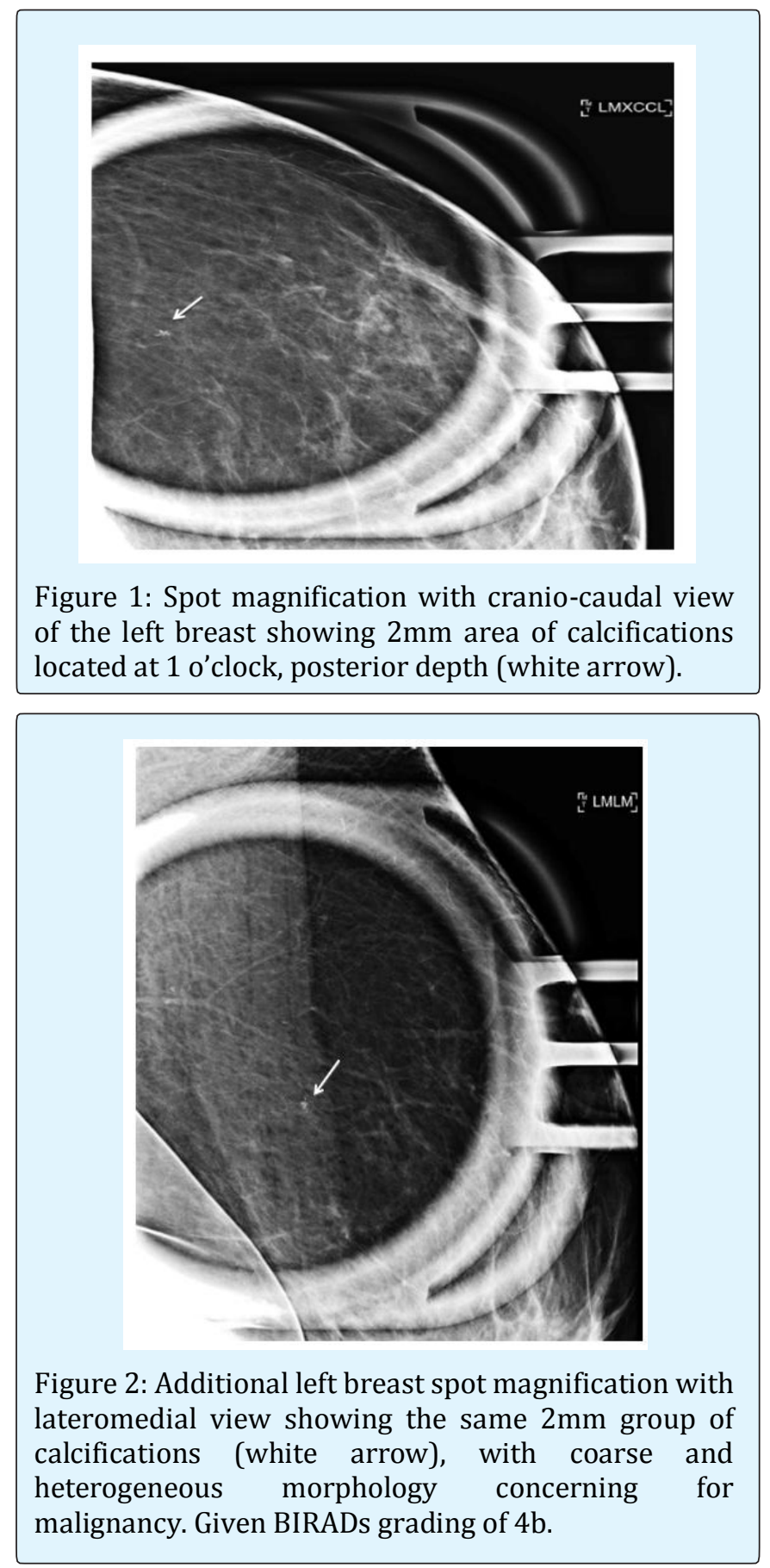

Results of the same day pathological analysis revealed a "benign breast tissue associated with osseous metaplasia" Figure 3. Results were communicated to the patient and no additional follow-ups were scheduled. The patient was advised to return to her normal annual mammographic screening schedule.

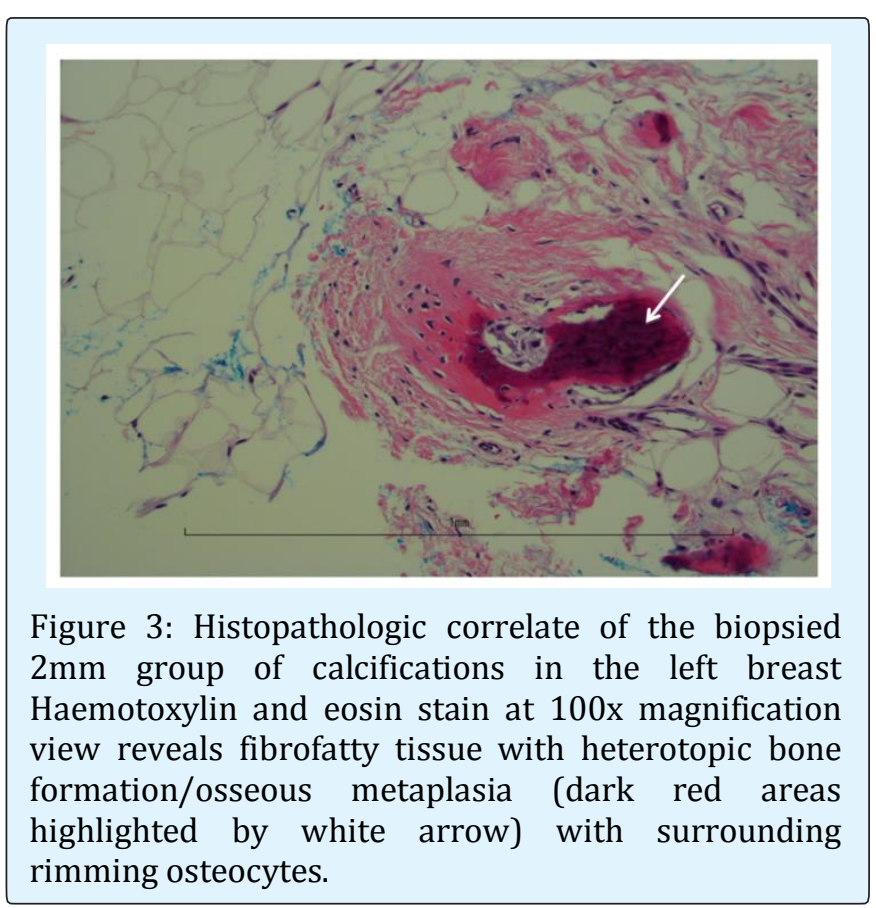

\section{Discussion}

Osseous metaplasia of the breast without other associated pathology is a very rare entity. It is defined the formation of extraosseous bone within the soft tissue secondary to the reversible transformation of one adult cell type into another type, which can occur within any location in the body, including the breast [1]. To our knowledge, there are only five other reported cases of osseous metaplasia of the breast without associated underlying pathology [2-4].

It has been documented that osseous metaplasia is a known etiology that can occur in many locations within the body, such as the endometrium, ovaries, thyroid, urinary bladder, thymus, and scar tissues, among others [5-7]. However, rarely, have there been reported instances of occurrence in the breast. Of the most commonly reported cases, osseous metaplasia of the breast usually occurs in the presence of underlying pathology, whether benign or malignant. Benign reported cases have been cited to develop under instances of trauma, hematoma, abscess, and within scar tissue formation. However, most commonly it develops in the setting of breast neoplasm such as malignant ductal 


\section{Clinical Radiology \& Imaging Journal}

carcinoma, metaplastic carcinoma of the breast, as well as malignant phyllodes tumor [8].

Even though no pathophysiology is known regarding specifically osseous metaplasia of the breast, in 1863, Virchow first postulated about the general pathophysiology of osseous metaplasia. He postulated that osteoblasts may arise from fibroblasts by metaplasia. He believed that osteoblasts were in fact, modified fibroblasts [9]. From this transformation, according to Gal-Gambos, et al. "the origin of metaplastic bone is like the development of normal bone. It may originate either directly from fibrous tissue or may be secondary to cartilage formation" [9].

In the breast, along similar lines of reasoning, it is possible that breast stromal fibroblasts may spontaneously undergo transformation into osteoblasts and produce non-neoplastic bony lesions within the normal breast stroma. In our patient, the osseous metaplasia may have been the result of a spontaneous transformation. However, it is also possible that she may have had non-reported minor trauma in the past, which resulted in local breast tissue inflammatory reactions and initiation of non differentiated "stem cells" or "reserve cells" which then resulted in the transformation of mesenchymal cells into osteoblasts and the production of osseous heterotopia in the same region [1].

Further, it may be possible that she had a latent foreign body reaction to the capsule material of the saline breast implant itself and this was the early manifestation. Latent reactions to breast silicone implantations, although rare, have been documented. They were common with first and second-generation silicone breast implants and appeared to correlate with the leakage of internal material, duration of implant in-situ, implant integrity, or implant degeneration. The documented time to dystrophic calcification formation was approximately 1122 years post implantation [10]. In a different study, the rates of osseous calcific formation within breast augmentation using injections of paraffin or silicone were generally detected at 12-17 years post injection [4]. This timeline fits well with our patient who had her saline breast implantation approximately 15 years prior and fits within the documented timeline for osseous formation.

\section{Conclusion}

Whatever the reason for calcific densities in the breast, identifying the finding and correctly establishing pathology is critical for optimal management. Identifying differences between osseous metaplasia and calcific deposition from dystrophic calcification or metastatic calcification is important in the clinical management and resulting patient prognosis. The former takes a benign course and the other a more involved treatment for the patient. However, when faced with calcifications in the setting of prior breast augmentation; it is pertinent to consider benign osseous metaplasia, along with more sinister etiologies within the differential considerations.

\section{References}

1. Christine MLS, Jefferson AA, Timperley WR, Watson N (1983) Osseous Metaplasia: a Late Pathological Finding within the Scar Tissue Formed Following Spinal Injury. Spinal Cord 21(6): 380-387.

2. Steinberg J, D'Alfonso T, Eisen C, Arleo EK (2016) Osseous Metaplasia of the Breast Diagnosed from Stereotactic Core Biopsy: A Rare Entity with Radiologic-Pathologic Correlation. The Breast Journal 22(4): 460-461.

3. Alyami H, Al-Osail E, Harbi S, Bu Bshait M (2018) Benign Osseous Metaplasia of the Breast: Case Report. Int J Surg Case Rep 44: 90-92.

4. Peters WJ, Pritzker KPH (1985) Massive Heterotopic Ossification in Breast Implant Capsules. Aesthetic Plastic Surgery 9(1): 43-45.

5. Bahceci M, Demirel LC (1996) Case report: Osseous metaplasia of the endometrium: a rare cause of infertility and its hysteroscopic management. Human Reproduction 11(11): 2537-2539.

6. Singh P, Kapur K, Singla S, Naz N (2011) Endometrial osseous metaplasia and mature bone formation with extramedullary hematopoiesis. J Hum Reprod Sci 4(1): 56-57.

7. Chun SJ, Hong R, Jung AK (2013) Osseous metaplasia with mature bone formation of the thyroid gland: Three case reports. Oncology Letters 6(4): 977-979.

8. Kijima Y, Umekita Y, Yoshinaka H, Owaki T, Sakamoto A, et al. (2006) A case of breast carcinoma with cartilaginous and osseous metaplasia. Breast Cancer 13(2): 214-219.

9. Gal-Gombos EC, Esserman LE, Poniecka AW, Odzer SL, Weisberg S, et al. (2002) Osseous Metaplasia of the Breast: Diagnosis with Stereotactic Core Biopsy. The Breast Journal 8(1): 50-52.

10. Fradinho N, Varanda A, Martins JB, Pedro AM (2015) Heterotopic Ossification in Breast Prosthesis. EPlasty 15: ic7. 